\title{
Nonlinear analysis of the pressure field in industrial buildings with curved metallic roofs due to the wind effect by FEM
}

\author{
J.J. del Coz Díaz ${ }^{1, *}$, P.J. García Nieto ${ }^{2}$, J.A. Vilán Vilán ${ }^{3}$, F.P. Alvarez Rabanal ${ }^{1}$, \\ A.Navarro Manso ${ }^{1}$ and M. Alonso Martínez ${ }^{1}$ \\ ${ }^{1}$ Department of Construction, GICONSIME Research Team, University of Oviedo, \\ Departmental Building 7, 33204 Gijón, Spain \\ ${ }^{2}$ Department of Mathematics, Faculty of Sciences, University of Oviedo, 33007 Oviedo, Spain \\ ${ }^{3}$ Department of Mechanical Engineering, University of Vigo, 36200 Vigo, Spain
}

\begin{abstract}
In this paper, an evaluation of distribution of the air pressure is determined throughout the laterally closed industrial buildings with curved metallic roofs due to the wind effect by the finite element method (FEM). The non-linearity is due to Reynolds-averaged Navier-Stokes (RANS) equations that govern the turbulent flow. The Navier-Stokes equations are non-linear partial differential equations and this non-linearity makes most problems difficult to solve and is part of the cause of turbulence. The RANS equations are time-averaged equations of motion for fluid flow. They are primarily used while dealing with turbulent flows. Turbulence is a highly complex physical phenomenon that is pervasive in flow problems of scientific and engineering concern like this one. In order to solve the RANS equations a two-equation model is used: the standard $k-\varepsilon$ model. The calculation has been carried out keeping in mind the following assumptions: turbulent flow, an exponential-like wind speed profile with a maximum velocity of $40 \mathrm{~m} / \mathrm{s}$ at $10 \mathrm{~m}$ reference height, and different heights of the building ranging from 6 to 10 meters. Finally, the forces and moments are determined on the cover, as well as the distribution of pressures on the same one, comparing the numerical results obtained with the Spanish CTE DB SE-AE, Spanish NBE AE-88 and European standard rules, giving place to the conclusions that are exposed in the study.
\end{abstract}

Keywords: Finite element modelling; Reynolds-averaged Navier-Stokes (RANS) equations; Standard $k-\varepsilon$ model; Buildings with curved metallic roofs; Numerical simulation

\section{Introduction}

The use of finite element method (FEM) [1-3] shows innumerable advantages of economical and practical order due, in the first place, to the cost that plays the realization of actual tests and, secondly, to the technical difficulty of the same ones, sine the elements object of the present study are big in size. The main aim of this paper is to determine, by FEM, the pressure distribution throughout the curved and laterally closed self-weighted metallic roofs on which the air wind falls horizontally [4].

The system of self-weighted metallic roofs constitutes an original alternative in the construction field (see Fig. 1). The metallic roof shells carry out a double function based

\footnotetext{
* Corresponding author. Tel.: +34-985-182042; fax: +34-985-182433.

E-mail address: juanjo@constru.uniovi.es (J. J. del Coz Díaz).
} 


\section{Highlights}

- Reliable results for pressure were obtained by using steady RANS-CFD simulations.

- We have determined the forces and moments on the cover with accurateness.

- A standard $k-\varepsilon$ model is integrated to investigate the wind effect by FEM. 
on the principle that the element of roof has to work like resistant element too: on the one hand, they act like arch beam and, on the other hand, like a building envelope.

In the majority of moderate speed flow structural problems some form of random variation of flow variables exists. The 'laminar' treatment is generally not applicable when such variations occur. Turbulent flow is defined as a flow with random variation of various flow quantities such as velocity, pressure and density. Turbulence is a property of a flow, not that of a fluid. Numerical solutions of the transient NavierStokes equations are sufficient to resolve the turbulent behaviour if an adequate fine mesh resolution and time increment are used. However, this requires extremely large computer resources and with present day computers a direct numerical simulation (DNS) is possible only at relatively low Reynolds numbers. Despite significant progress in understanding turbulent behaviour during the last century, the modelling of turbulence is still an unresolved problem and will remain so for the foreseeable future [5-10].

In this work, we have proceeded to calculate the pressure coefficient on a laterally closed industrial building with a curved metallic roof $[4,11]$. Since this problem is highly turbulent, we have had to integrate the Reynolds-averaged Navier-Stokes (RANS) equations inside the $k-\varepsilon$ model of turbulence by the finite element method (FEM). The $k-\varepsilon$ model is one of the most common turbulence models. It is a two equation model since it includes two extra transport equations to represent the turbulent properties of the flow. This allows a two equation model to account for history effects like convection and diffusion of turbulent energy. The first transported variable is turbulent kinetic energy $k$. The second transported variable in this case is the turbulent dissipation $\varepsilon$. It is the variable that determines the scale of the turbulence, whereas the first variable, $k$, determines the energy in the turbulence.

Fig. 1. Actual view of a curved self-weighted metallic roof.

\section{Mathematical modelling of turbulence}

Turbulence is a highly complex physical phenomenon that is dominant in flow problems of scientific and engineering concern. A simple, precise definition of turbulence is difficult though the phenomenon is often associated with the ideas of randomness, disorder, and chaos.

Turbulence is defined as an "irregular flow condition showing random variations with respect to both time and space coordinates with discernible statistical properties". A turbulent flow is a $[12,13]$ :

- Highly non-linear flow process.

- Highly diffusive flow.

- Three-dimensional flow.

- Flow with multiple length and time scales.

- Time-dependent (stochastic) phenomenon with identifiable statistical properties. 
Turbulence is one of the unsolved problems in physics, especially in the sense that universally applicable mathematical models of the phenomenon are not available. Our interest here will therefore focus on the modelling and simulation of turbulence from an engineering point of view. This approach implies that the detailed resolution of a turbulent flow will be eliminated in favour of some type of averaged flow description. Turbulence effects will enter the flow description via a model that is typically based on a combination of theory and experiment.

Next, the equations for the mean flow will be described followed by an outline of various types of turbulence models.

\subsection{Governing equations}

A majority of researchers accept the notion that, in principle, the Navier-Stokes equations are capable of fully describing a turbulent flow. The natural question that follows this premise is if the Navier-Stokes equations are valid, why not solve them directly via a numerical method called Direct Numerical Simulation (DNS) to obtain the needed turbulent solution?

The use of DNS is still possible only for rather simple flow cases at low Reynolds numbers. The restrictions of DNS are obvious since [12-16]:

- The number of grid points needed for sufficient spatial resolution varies as $\operatorname{Re}^{9 / 4}$ (characteristic eddy length is $0.001 L$, where $L$ is the characteristic flow dimension).

- The CPU-time varies as $\mathrm{Re}^{3}$.

The standard alternative to the DNS approach involves the solution to some form of averaged Navier-Stokes equations. In most flow problems of interest it is the mean flow that is of most concern, with the turbulent fluctuations only being important in how they influence the mean flow evolution. By performing a suitable average on the instantaneous Navier-Stokes equations, a standard mean flow problem can be derived where the effects of the turbulence are relegated to a few terms that can be modelled. This approach forms the basis for most of our current computational work.

To outline this approach, let the instantaneous fluid velocity and pressure fields be expressed as the sum of a mean and fluctuating component. That is $[13,15,16]$ :

$$
\begin{gathered}
u_{i}=U_{i}+u_{i}^{\prime} \\
p=P+P^{\prime}
\end{gathered}
$$

Substituting these definitions into the incompressible, viscous flow equation produces the RANS equations $[13,15,16]$ :

$$
\begin{gathered}
\frac{\partial U_{i}}{\partial x_{i}}=0 \\
\rho\left(\frac{\partial U_{i}}{\partial t}+U_{j} \frac{\partial U_{i}}{\partial x_{j}}\right)=-\frac{\partial P}{\partial x_{i}}+\frac{\partial}{\partial x_{j}}\left[\mu\left(\frac{\partial U_{i}}{\partial x_{j}}+\frac{\partial U_{j}}{\partial x_{i}}\right)-\overline{\rho u_{i}^{\prime} u_{j}^{\prime}}\right]+\rho g_{i}
\end{gathered}
$$

Eqs. (3) and (4) describe the behaviour of the mean fluid velocity and pressure fields. The extra term that appears in Eq. (4) is often termed the Reynolds stress and represents 
the effects of the turbulent velocity fluctuations on the mean flow. Note that those second-order moments from the nine components of a second-order tensor, though symmetry considerations reduce this to a total of six independent unknowns.

\subsection{General turbulence models}

The completion of the mean flow boundary value problem described by Eqs. (3) and (4) requires that six additional equations for the Reynolds stresses be provided. This specification constitutes a turbulence model. There are an enormous variety of turbulence models, ranging in complexity from simple algebraic statements to descriptions involving multiple, non-linear partial differential equations. Unfortunately, there is no universal method of classification for such models which adds greatly to the confusion within the field. Here we follow the classification scheme by Ferziger and Peric $[13,17]$, which groups turbulence models according to the following labels:

- Correlation.

- Integral methods.

- One point closure.

- Two point closure.

- Large eddy simulation.

- Direct numerical simulation.

\subsection{One point closure models}

The majority of computational work, especially for industrial applications, has relied on some form of the one point closure model. A classification scheme for these models is shown in Fig. 2 [13]:

Fig. 2. A classification scheme for the turbulent one point closure models.

\subsection{Eddy viscosity models}

Of the two major branches shown before, we will concentrate on the so-called eddy viscosity models. The Reynolds stress models (RSM), generally more sophisticated than the eddy viscosity approach, lead to large systems of partial differential equations and a large number of empirical parameters. It is important to note that eddy viscosity models are based on one major assumption: the Boussinesq hypothesis. By analogy with the molecular diffusion of momentum, the Boussinesq hypothesis relates the turbulent momentum transport to the gradients of the mean velocity field. The Reynolds stresses in (4) are then expressed by [13-18]:

$$
-\overline{\rho u_{i}^{\prime} u_{j}^{\prime}}=\mu_{T}\left(\frac{\partial U_{i}}{\partial x_{j}}+\frac{\partial U_{j}}{\partial x_{i}}\right)
$$

where $\mu_{T}$ is the eddy viscosity. Unlike the molecular viscosity, $\mu$, which is a fluid property, the eddy viscosity is a local property of the flow.

When the definition in Eq. (5) is substituted into the momentum equation then the equations for the mean flow become: 


$$
\begin{gathered}
\frac{\partial U_{i}}{\partial x_{i}}=0 \\
\rho\left(\frac{\partial U_{i}}{\partial t}+U_{j} \frac{\partial U_{i}}{\partial x_{j}}\right)=-\frac{\partial P}{\partial x_{i}}+\frac{\partial}{\partial x_{j}}\left[\left(\mu+\mu_{T}\right)\left(\frac{\partial U_{i}}{\partial x_{j}}+\frac{\partial U_{j}}{\partial x_{i}}\right)-\rho u_{i}^{\prime} u_{j}^{\prime}\right]+\rho g_{i}
\end{gathered}
$$

Once the form of the eddy viscosity is specified then the mean flow can be solved in the same manner as a laminar flow since the equations are the same except for an augmented viscosity. Though the turbulent flow problem has been reduced to a familiar set of partial differential equations, there remains the nontrivial task of specifying how the eddy viscosity varies with the flow field. Scaling arguments show that the eddy viscosity is proportional to a characteristic eddy velocity, $u_{e}$, and an eddy length, $l_{e}$. Therefore it is verified that [13-16]:

$$
\mu_{T} \propto \rho u_{e} l_{e}
$$

\subsubsection{Zero-equation model}

The type of turbulence model used is determined by the number of equations used to specify the variation of the previous variables $u_{e}$ and $l_{e}$. This model calculates the eddy viscosity by an algebraic prescription of $u_{e}$ and $l_{e}$ based on Prandtl's mixing length, which specifies $l_{e}$ to be the length scale across which turbulent mixing takes place. Prandtl gave the characteristic velocity $u_{e}$ to be [13-16]:

$$
\begin{aligned}
& u_{e}=l_{e}\left[\left(\frac{\partial U_{i}}{\partial x_{j}}+\frac{\partial U_{j}}{\partial x_{i}}\right) \frac{\partial U_{i}}{\partial x_{j}}\right]^{1 / 2} \\
& \mu_{T}=\rho l_{e}^{2}\left[\left(\frac{\partial U_{i}}{\partial x_{j}}+\frac{\partial U_{j}}{\partial x_{i}}\right) \frac{\partial U_{i}}{\partial x_{j}}\right]^{1 / 2}
\end{aligned}
$$

For a number of geometrically simple flows, the variation of the mixing length, $l_{e}$, is well known and can be evaluated via simple formulas. Eq. (10) then allows to be derived and the turbulence model completed. Flows that are amenable to such treatment include pipe and channel flows, jets, wakes, and boundary layers.

\subsubsection{One-equation model}

The algebraic specification of $u_{e}$ can be replaced with a more generally applicable transport equation. Since the characteristic velocity $u_{e}$ is proportional to the square root of the turbulent kinetic energy, $k$, then:

$$
u_{e} \propto k^{1 / 2}
$$

so that

$$
\mu_{T} \propto \rho k^{1 / 2} l_{e}
$$

A partial differential equation for $k$ can be derived from the Navier-Stokes equation and is given by $[13-16,19,20]$ : 


$$
\rho\left(\frac{\partial k}{\partial t}+U_{j} \frac{\partial k}{\partial x_{j}}\right)=\frac{\partial}{\partial x_{j}}\left(\frac{\mu_{T}}{\sigma_{k}} \frac{\partial k}{\partial x_{j}}\right)+\rho G-\rho \varepsilon
$$

where $G$ is a generation term, $\varepsilon$ is the turbulent dissipation, and $\sigma_{k}$ is a constant.

\subsubsection{Two-equation model}

A natural evolution of the one equation model involved the replacement of the algebraic relation for mixing length with a second transport equation. Dimensional arguments lead to the proportionality:

$$
l_{e} \propto \frac{k^{3 / 2}}{\varepsilon}
$$

where $\varepsilon$ is the turbulent dissipation. Substituting (14) and (11) into Eq. (8) produces the proportionality:

$$
\mu_{T} \propto \rho \frac{k^{2}}{\varepsilon}
$$

or the Kolmogorov-Prandtl relation [13-16, 19, 20]:

$$
\mu_{T}=C_{\mu} \rho \frac{k^{2}}{\varepsilon}
$$

which relates the eddy viscosity directly to the turbulent variables, $k$ and $\varepsilon$.

\subsubsection{1. $k-\varepsilon$ model}

The turbulent dissipation $\varepsilon$ is described by an equation of similar form to Eq. (13) for $k$ [13-16]:

$$
\rho\left(\frac{\partial \varepsilon}{\partial t}+U_{j} \frac{\partial \varepsilon}{\partial x_{j}}\right)=\frac{\partial}{\partial x_{j}}\left(\frac{\mu_{T}}{\sigma_{\varepsilon}} \frac{\partial \varepsilon}{\partial x_{j}}\right)+\rho \frac{\varepsilon}{k}\left(c_{1} G-c_{2} \varepsilon\right)
$$

where $G$ is again a shear generation term and $c_{1}$ and $c_{2}$ are empirically derived constants as are $\sigma_{k}$ and $\sigma_{g}$. The two-equation $k-\varepsilon$ model described by Eqs. (13) and (17) can be used in conjunction with the mean flow equations and the definition of $\mu_{T}$ given by Eq. (16), to arrive at a continuum description of turbulent flow. The equation set is highly non-linear, with a strong coupling between the various transport equations. The $k-\varepsilon$ model is far from universal and has a number of weaknesses, though it remains one of the most heavily used methods for turbulent flow simulations.

\section{Implementation of the standard model}

The turbulent averaging process is introduced in order to obtain the laws of motion for the mean, time-averaged, turbulent quantities $[12,13]$. This time averaging is to be defined in such a way as to remove the influence of the turbulent fluctuations while not destroying the time dependence associated with other time-dependent phenomena with time scales distinct from those of turbulence. For incompressible flows that are turbulent the use of the three dimensional equivalent of Navier-Stokes equations would be too expensive for engineering design calculations. For most practical calculations, the mean motion is of primary interest. This can be obtained by first averaging the 
equations over a small time $T$. This process produces the time-averaged governing equations: the RANS equations.

In summary, in the framework of eddy viscosity models, the hydrodynamic behaviour of a turbulent incompressible fluid is governed by the RANS equations for the velocity $\vec{u}$ and pressure $p$ :

$$
\frac{\partial \vec{u}}{\partial t}+\vec{u} \cdot \nabla \vec{u}=-\nabla p+\nabla \cdot\left(\left(v+v_{T}\right)\left[\nabla \vec{u}+\nabla \vec{u}^{T}\right]\right), \quad \nabla \cdot \vec{u}=0
$$

where $v$ depends only on the physical properties of the fluid, while $v_{T}$ is the turbulent eddy viscosity which is supposed to emulate the effect of unresolved velocity fluctuations $\vec{u}^{\prime}$.

If the standard $k-\varepsilon$ model is employed, then $v_{T}=C_{\mu} \frac{k^{2}}{\varepsilon}$, where $k$ is the turbulent kinetic energy and $\varepsilon$ is the dissipation rate. Hence, the above PDE system is to be complemented by two additional convection-diffusion-reaction equations for computation of $k$ and $\varepsilon$ :

$$
\begin{aligned}
& \frac{\partial k}{\partial t}+\nabla \cdot\left(k \vec{u}-\frac{v_{T}}{\sigma_{k}} \nabla k\right)=P_{k}-\varepsilon \\
& \frac{\partial \varepsilon}{\partial t}+\nabla \cdot\left(\varepsilon \vec{u}-\frac{v_{T}}{\sigma_{\varepsilon}} \nabla \varepsilon\right)=\frac{\varepsilon}{k}\left(C_{1} P_{k}-C_{2} \varepsilon\right)
\end{aligned}
$$

where $P_{k}=\frac{v_{T}}{2}\left|\nabla \vec{u}+\nabla \vec{u}^{T}\right|^{2}$ and $\varepsilon$ are responsible for production and dissipation of turbulent kinetic energy, respectively. The default values of the involved empirical constants are as follows: $C_{\mu}=0.09, C_{1}=1.44, C_{2}=1.92, \sigma_{k}=1.0, \sigma_{\varepsilon}=1.3$. Eqs. (18)(20) are to be endowed with appropriate initial/boundary conditions which will be discussed later.

\subsection{Iterative solution strategy}

The Navier-Stokes equations are an example of a non-linear mixed hyperbolic-parabolic system with non-linear hyperbolic convection terms $\vec{u} \cdot \nabla \vec{u}$ and a linear elliptic viscous terms $-v \Delta \vec{u}$. The discretization is space is performed by an unstructured grid finite element method. The incompressible Navier-Stokes equations are discretized using the nonconforming $\tilde{Q}_{1} / Q_{0}$ element pair, whereas standard $Q_{1}$ elements are employed for $k$ and $\varepsilon$. After an implicit time discretization by the Crank-Nicolson or backward Euler methods, the nodal values of $(\vec{v}, p)$ and $(k, \varepsilon)$ are updated in a segregated fashion within an outer iteration loop.

For our purposes, it is worthwhile to introduce an auxiliary parameter $\gamma=\varepsilon / k$, which makes it possible to decouple the transport equations (19) and (20) as follows [21-23]:

$$
\frac{\partial k}{\partial t}+\nabla \cdot\left(k \vec{u}-\frac{v_{T}}{\sigma_{k}} \nabla k\right)+\gamma k=P_{k}
$$




$$
\frac{\partial \varepsilon}{\partial t}+\nabla \cdot\left(\varepsilon \vec{u}-\frac{v_{T}}{\sigma_{\varepsilon}} \nabla \varepsilon\right)+C_{2} \gamma \varepsilon=\gamma C_{1} P_{k}
$$

This representation provides a positivity-preserving linearization of the sink terms, whereby the parameters $v_{T}$ and $\gamma$ evaluated using the solution from the previous outer iteration $[21,22,24]$. The iterative solution process is based on the following hierarchy of nested loops (see Fig. 3):

Fig. 3. Hierarchy of nested loops for the iterative solution process.

At each time step (one $n$-loop iteration), the governing equations are solved repeatedly within the outer $k$-loop which contains the two subordinate $l$-loops responsible for the coupling of variables within the corresponding subproblem. The embedded $m$-loops correspond to iterative flux/defect correction for the envolved convectiondiffusion operators. Flux limiters of TVD type are activated in the vicinity of steep gradients, where nonlinear artificial diffusion is required to suppress non-physical undershoots and overshoots. In the case of an implicit time discretization, subproblem (21)-(22) leads to a sequence of algebraic systems of the form [24]:

$$
A\left(\vec{u}^{(k)}, \gamma^{(l)}, v_{T}^{(k)}\right) \Delta u^{(m+1)}=r^{(m)}, u^{(m+1)}=u^{(m)}+\omega \Delta u^{(m+1)}
$$

where $r^{(m)}$ is the defect vector and the superscripts refer to the loop in which the corresponding variable is updated. The predicted values $k^{(l+1)}$ and $\varepsilon^{(l+1)}$ are used to recompute the linearization parameter $\gamma^{(l+1)}$ for the next outer iteration (if any). The associated eddy viscosity $v_{T}$ is bounded from below by a certain fraction of laminar viscosity $0<v_{\min } \leq v$ and from above by $v_{\max }=l_{\max } \sqrt{k}$, where $l_{\max }$ is the maximum admissible mixing length (the size of the largest eddies, e.g., the width of the domain). Specifically, we define the limited mixing length $l_{*}$ as:

$$
l_{*}=\left\{\begin{array}{cc}
C_{\mu} \frac{k^{3 / 2}}{\varepsilon} \quad \text { if } \quad C_{\mu} k^{3 / 2}<\varepsilon l_{\max } \\
l_{\max } \text { otherwise }
\end{array}\right.
$$

and calculate the turbulent eddy viscosity $v_{T}$ from the formula:

$$
v_{T}=\max \left\{v_{\min }, l_{*} \sqrt{k}\right\}
$$

The resulting value of (25) is used to update the linearization parameter:

$$
\gamma=C_{\mu} \frac{k}{v_{T}}
$$

The above representation of $v_{T}$ and $\gamma$ makes it possible to preclude division by zero and obtain bounded nonnegative coefficients without manipulating the actual values of $k$ and $\varepsilon$.

\section{Hypotheses of this problem}

For the fluid phase, we do the following assumptions: 
- The flow is incompressible and subsonic $[4,11,14,17,18]$ :

$$
\mathrm{Ma}=\frac{v_{r e f}}{c}=\frac{40 m / s}{340 m / s}=0.118<<0.3 \Rightarrow \nabla \cdot \vec{v}=0
$$

- Standard $k-\varepsilon$ turbulence modelling is used since the flow is highly turbulent $[14,17,18]$ :

$$
\operatorname{Re}=\frac{v_{r e f} \cdot L_{r e f}}{v_{r e f}}=\frac{40 \mathrm{~m} / \mathrm{s} \cdot 8 \mathrm{~m}}{1.34 \times 10^{-9} \mathrm{~m}^{2} / \mathrm{s}}=23.89 \times 10^{10}
$$

\subsection{Wind speed profile}

The variation of the wind speed with respect to the height can be evaluated in a first approximation by means of the following potential-type expression $[4,11,14,17,18]$ :

where:

$$
v_{\text {wind }}=v_{\text {ref }} \cdot\left(\frac{h}{h_{\text {ref }}}\right)^{n}
$$

- $v_{\text {wind }}=$ is the wind speed to the height $h$ with respect to the ground in the $X$ direction.

- $\quad v_{r e f}=$ is the wind speed known to the reference height $h_{r e f}$. In our case we have taken a value of $v_{\text {ref }}=40 \mathrm{~m} / \mathrm{s}$ for $h_{\text {ref }}=10 \mathrm{~m}$.

- $h=$ is the height to which we wish to estimate the wind speed.

- $h_{r e f}=$ is the reference height.

- $n=$ value that depends on the existing roughness in the place (location).

Next, Table 1 shows an estimation of the value of $n$ for different grounds:

Table 1

Value of $n$ for different types of ground.

In this study, we have taken $n=0.1$ corresponding to a smooth-type ground $[4,11,14$, $17,18]$.

\section{Geometrical model and boundary conditions}

\subsection{Geometrical model}

On the one hand, it is understood for domain the whole of space points where the objective functions must verify the differential equations of the model. In a boundary value problem, the values of some degrees of freedom in the boundary of domain are known. For this problem of external flow around a body (see Fig. 4), the domain is an air volume that does not contain the interior of the building. This air box is divided in finite elements with the characteristic properties of the air. This process is called meshing. All the volume of the box is meshed excepting but the space occupied by the industrial building. The size of the air box in the models, where the flow is studied around the body, is submerged in a stream, so that the position of the body inside the 
domain depends on the size and shape of the body as well as the fluid-dynamical characteristics of the problem.

Fig. 4. Dimensions of the roof: length $L=45 \mathrm{~m}$; width $W=30 \mathrm{~m}$; height $H=8 \mathrm{~m}$; arrow and $f=3.6 \mathrm{~m}$.

\subsection{Initial conditions}

It is rather difficult to devise a reasonable initial guess for a steady-state simulation or proper initial conditions for a dynamic one. If the velocity field is initialized by zero, it takes the flow some time to become fully turbulent. Therefore, we activate the $k-\varepsilon$ model at a certain time $t_{*}>0$ after the startup. During the laminar initial phase $\left(t \leq t_{*}\right)$, a constant effective viscosity $v_{0}=O(v)$ is prescribed. The values to be assigned to $k$ and $\varepsilon$ at $t=t_{*}$ are uniquely defined by the choice of $v_{0}$ and of the default mixing length $l_{0} \in\left[l_{\min }, l_{\max }\right]$ where the threshold parameter $l_{\min }$ corresponds to the size of the smallest admissible eddies. Thus, we have:

$$
k_{0}=\left(\frac{v_{0}}{l_{0}}\right)^{2}, \quad \varepsilon_{0}=C_{\mu} \frac{k_{0}^{3 / 2}}{l_{0}} \text { at } t \leq t_{*}
$$

Alternatively, the initial guess of $k$ and $\varepsilon$ can be estimated by means of a zero-equation (mixing length) turbulence model or computed using an extension of the inflow or wall boundary conditions (see below) into the interior of the computational domain.

\subsection{Implementation of boundary conditions}

The adopted boundary conditions in this problem are the following ones $[4,11,13-16$, 19, 20]:

- Inlet: $U=$ wind speed profile (indicated previously in Eq. 11); $V, W=0$ (components of wind speed in $Y$ and $Z$ directions are zero); $k=c_{b c}|\vec{u}|^{2}$, $\varepsilon=C_{\mu} k^{3 / 2} / l_{0}$ on the inflow boundary $\Gamma_{i n}$, where $c_{b c} \in[0.003,0.01]$ is an empirical constant and $|\vec{u}|=\sqrt{\vec{u} \cdot \vec{u}}$ is the Euclidean norm of the velocity.

- Outlet: Relative pressure $p=0$. At the outlet $\Gamma_{\text {out }}$, the normal gradients of all variables are set equal to zero, which corresponds to the Neumann boundary condition: $\partial \vec{v} /\left.\partial n\right|_{\text {outlet }}=0$ (Homogeneous Neumann boundary condition for the velocity vector); $\vec{n} \cdot \nabla k=0$, that is to say, $\partial k /\left.\partial n\right|_{\text {outlet }}=0 ; \vec{n} \cdot \nabla \varepsilon=0$, that is to say, $\partial \varepsilon /\left.\partial n\right|_{\text {outlet }}=0$ (homogeneous Neumann boundary condition for the turbulent unknowns).

- Solid walls: The non-slip condition $(U, V, W=0)$ is the appropriate condition for the velocity components at solid walls $\Gamma_{w}$ (roof and lateral walls). Close to the wall the flow is influenced by viscous effects and does not depend on free stream parameters. The implementation of wall boundary condition in turbulent flows is considered to be in the log-law region of a turbulent boundary layer [15, 16]. 
The two-dimensional model has been solved with a reference velocity of $v_{\text {ref }}=40 \mathrm{~m} / \mathrm{s}$.

\subsection{Wall functions}

To complete the problem statement, we still need to prescribe the tangential stress as well as the boundary conditions for $k$ and $\varepsilon$ on $\Gamma_{w}$. Note that the equations of the $k-\varepsilon$ model are invalid in the vicinity of the wall where the Reynolds number is rather low and viscous effects are dominant. In order to avoid the need for resolution of strong velocity gradients, wall functions are typically applied at an intenal boundary $\Gamma_{y}$ located at a distance $y$ from the solid wall $\Gamma_{w}$ :

$$
\vec{n} \cdot\left[\nabla \vec{u}+\nabla \vec{u}^{T}\right]=-\frac{u_{\tau}^{2}}{v_{T}} \frac{\vec{u}}{|\vec{u}|}, k=\frac{u_{\tau}^{2}}{\sqrt{C_{\mu}}}, \varepsilon=\frac{u_{\tau}^{3}}{\kappa y} \text { on } \Gamma_{y}
$$

where $\kappa=0.41$ is the von Kármán constant. The above mentioned free-slip condition is also to be imposed on $\Gamma_{y}$ rather than on $\Gamma_{w}$. Therefore, $\vec{u}$ is the tangential velocity which can be used to compute the friction velocity $u_{\tau}$ from the nonlinear equation:

$$
\frac{|\vec{u}|}{u_{\tau}}=\frac{1}{\kappa} \log y^{+}+\beta,
$$

valid in the logarithmic layer, where the local Reynolds number $y^{+}=\frac{u_{\tau} y}{v}$ is in the range $11.06 \leq y^{+} \leq 300$. The empirical constant $\beta=5.2$ for smooth walls.

Strictly speaking, a boundary layer of width $y$ should be removed from the computational domain $\Omega$. However, it is supposed to be very thin, so that the equations can be solved in the whole domain $\Omega$ with wall functions prescribed on the boundary part $\Gamma_{w}$ rather than on $\Gamma_{y}$. Since the choice of $y$ is rather arbitrary, it is worthwhile to define the boundary layer width by fixing $y_{+}$, as proposed in $[25,26]$. The implicitly defined $y=v y_{+} / u_{\tau}$ is assumed to be the point where the logarithmic layer meets the viscous layer so that $y_{+}$satisfies (32) as well as the linear relation $y_{+}$. The corresponding parameter value $y_{+}^{*}$ is given by:

$$
y_{+}^{*}=\frac{1}{\kappa} \log y_{+}^{*}+\beta \approx 11.06 \text { on } \Gamma_{y}
$$

The use of $y_{+}^{*}$ in the wall laws (31)-(33) yields an explicit relation for the friction velocity $u_{\tau}$ which is required to evaluate the tangential stress for the momentum equations $[15,16,24]$ :

$$
\vec{n} \cdot\left[\nabla \vec{u}+\nabla \vec{u}^{T}\right]=-\frac{u_{\tau}^{*}}{v_{T}} \frac{\vec{u}}{y_{+}^{*}} \text { where } u_{\tau}^{*}=\max \left\{C_{\mu}^{0.25} \sqrt{k}, \frac{|\vec{u}|}{y_{+}^{*}}\right\}
$$

This expression provides a natural boundary condition for the tangential velocity:

$$
\int_{\Gamma_{w}} v_{T}\left(\vec{n} \cdot\left[\nabla \vec{u}+\nabla \vec{u}^{T}\right] \cdot \vec{w}\right) d s=-\int_{\Gamma_{w}} \frac{u_{\tau}^{*}}{y_{+}^{*}}(\vec{u} \cdot \vec{w}) d s
$$

Due to (31), the boundary value of the turbulent eddy viscosity is proportional to $v$ : 


$$
v_{T}=C_{\mu} \frac{k^{2}}{\varepsilon}=\kappa u_{\tau} y=\kappa y_{+}^{*} \nu
$$

Of course, the above relation is satisfied automatically if the boundary conditions for $k$ and $\varepsilon$ are implemented in the strong sense as proposed in [23, 24]. However, the use of Dirichlet boundary conditions means that the boundary values of $k$ and $\varepsilon$ depend solely on the friction velocity $u_{\tau}=|\vec{u}| / y_{+}^{*}$ which is proportional to the flow velocity at the wall. This results in a one-way coupling of the boundary conditions which is rather unrealistic. In order to let $k$ and $\varepsilon$ 'float' and influence the momentum equations via (34)-(35), the wall boundary conditions should be implemented in a weak sense. To this end, let us compute the boundary values of $v_{T}$ from Eq. (36) and invoke (31) to retrieve the normal derivatives of $k$ and $\varepsilon$ follows:

$$
\vec{n} \cdot \nabla k=-\frac{\partial k}{\partial y}=0, \quad \vec{n} \cdot \nabla \varepsilon=-\frac{\partial \varepsilon}{\partial y}=\frac{u_{\tau}^{3}}{\kappa y^{2}}=\frac{1}{v_{T}} \frac{u_{\tau}^{5}}{y_{+} v}
$$

These natural boundary conditions are to be plugged into the surface integrals resulting from integration by parts in the variational formulation for Eqs. (21) and (22):

$$
\int_{\Gamma_{w}} \frac{v_{T}}{\sigma_{k}}(\vec{n} \cdot \nabla k) w d s=0, \int_{\Gamma_{w}} \frac{v_{T}}{\sigma_{\varepsilon}}(\vec{n} \cdot \nabla \varepsilon) w d s=\int_{\Gamma_{w}} \frac{1}{\sigma_{\varepsilon}} \frac{u_{\tau}^{5}}{y_{+} v} w d s
$$

Furthermore, it is commonly assumed that $P_{k}=\varepsilon$ in the wall region, so that the correct boundary value of the production term must be computed from:

$$
P_{k}=\frac{u_{\tau}^{3}}{\kappa y}=\frac{u_{\tau}^{3}}{v_{T}} \frac{|\vec{u}|}{y_{+}^{*}}, \text { where } u_{\tau}=C_{\mu}^{0.25} \sqrt{k}
$$

The above implementation of wall functions is largely based on the publication of Grotjans and Menter [25] which should be consulted for further algorithmic details.

Therefore, in the near-wall regions [13-16, 25-27]:

- The use of Eqs. (13) and (17) implies that $\mu_{T}>>\mu$ (high Reynolds $k-\varepsilon$ model).

- This is invalid close to a solid wall where the turbulent fluctuations are suppressed due to the presence of the viscous sublayer.

- Therefore, adjacent to walls, special wall functions are introduced that assume a log-dependence of the tangential velocity on the normal coordinate so that the production of $k$ is equal to the dissipation in the log-law region.

- This is equivalent to introduce a mixing-length eddy viscosity formulation adjacent to a wall.

- The use of the special wall functions provides boundary conditions on $k$ and $\varepsilon$ away from the wall.

\section{Finite element analysis and results}

In order to simulate the turbulent fluid flow, FLUID 141 element was used in this study $[5,13,28]$. This element is defined by three nodes (triangle) or four nodes (quadrilateral) as well as by isotropic material properties (see Fig. 5).

In this element, the velocities are obtained from the conservation of momentum principle, and the pressure is obtained from the conservation of mass principle. A 
segregated sequential solver algorithm is used; that is, the matrix system derived from the finite element discretization of the governing equation for each degree of freedom is solved separately. The flow problem is nonlinear and the governing equations are coupled together. The sequential solution of all the governing equations, combined with the update of pressure-dependent properties, constitutes a global iteration. The number of global iterations required to achieve a converged solution may vary considerably, depending on the size and stability of the problem.

Fig. 5. Finite element FLUID 141 [28] used in the numerical simulation of laterally closed industrial buildings with curved metallic roofs by FEM.

The two-dimensional model has been solved with a reference velocity of $v_{\text {ref }}=40 \mathrm{~m} / \mathrm{s}$. Note that the negative pressure drop in the upper face of the roof ranges from $-2,800 \mathrm{~Pa}$ to $-200 \mathrm{~Pa}$, a constant positive pressure in the upstream lateral wall of about $815 \mathrm{~Pa}$ and a constant negative pressure in the downstream lateral wall of about $85 \mathrm{~Pa}$. Fig. 6a shows the pressure contour lines for the analyzed roof. Velocities in the building ranges from $95 \mathrm{~m} / \mathrm{s}$ to $0 \mathrm{~m} / \mathrm{s}$, with an important gradient in the transition from the upstream lateral wall to the roof, as it is shown in Fig. 6b. Finally, Figs. 6c and 6d show the contour lines for the turbulent kinetic energy and turbulent dissipation rate, respectively, for the same reference velocity.

Once we have solved the numerical model, it is proceeded to do the calculation of the pressure coefficient for the closed building analyzed. This coefficient $[14,18]$ is defined as $C_{P}=\left(P-P_{0}\right) /\left(\rho \cdot v^{2} / 2\right)$, where: $P$ is the pressure on the roof's surface, $P_{0}$ is the pressure of the non-disturbed stream, $v$ is the wind speed of the non-disturbed stream at the corresponding height and $\rho$ is the air density.

Fig. 6. Numerical contour lines: (a) pressure (Pa) for $v_{r e f}=40 \mathrm{~m} / \mathrm{s}$; (b) velocity $(\mathrm{m} / \mathrm{s}$ ) for $v_{\text {ref }}=40 \mathrm{~m} / \mathrm{s}$; (c) turbulent kinetic energy, $k(\mathrm{~J})$ and (d) turbulent dissipation rate, $\varepsilon$ $(\mathrm{J})$.

Comparing the pressure coefficients estimated by the Spanish and European rules [4, 11] with this one calculated by the finite element method (FEM), Fig. 7 is obtained. As it is observed in this figure, the agreement between European and CTE rules is quite good. Similarly, there is a good agreement between the old Spanish rule and the FEM results. The finite element results show that in the transition from the upstream lateral wall to the roof there is an important suction. The other previous rules do not take into account this phenomenon. Finally, from the structural point of view, the most secure rule is the old Spanish rule, according to the FEM results.

Fig. 7. Pressure coefficient $C_{P}$ obtained by FEM and the estimated ones by the Standard rules $[4,11]$. 


\section{Conclusions}

A computational procedure has been developed based on the general-purpose finite element code ANSYS-FLOTRAN [4, 28], for modelling and simulating the air pressure on the laterally closed industrial buildings with curved metallic roofs due to the wind effect. The findings of this study suggest that it may be possible to devise a practical procedure for stabilizing a self-weighted metallic roof model by using a computational approach.

Turbulence plays an important role in many engineering process (fluid flow, mass and heat transfer, chemical reactions, etc.) which are dominated by convective transport. Since the direct numerical simulation (DNS) of turbulent flows is still prohibitively expensive, eddy viscosity models based on the Reynolds averaged Navier-Stokes (RANS) equations are commonly employed in CFD codes. One of the most popular ones is the standard $k-\varepsilon$ model which has been in use since the 1970s. However, its practical implementation and, especially, the near-wall treatment has always been some somewhat of a mystery. A positivity-preserving discretization of the troublesome convective terms is an important prerequisite for the robustness of the numerical algorithm. This paper presents a detailed numerical study of the $k-\varepsilon$ model using algebraic flux correction to enforce the positivity constraint.

The problem was solved in a workstation computer with a CPU Intel Xeon 5140 @ 2.33 GHz, 24 GB RAM memory and 4 TB hard disk. The total CPU time employed for each simulation was 1,845 seconds and the total number of iterations in order to get the convergence was about 7,100.

It is clear that the finite element solution will solve only the selected mathematical model and that all assumptions in this model will be reflected in the predicted response [1-3]. We can not expect any more information in the prediction of physical phenomena than the information contained in the mathematical model. Hence the choice of an appropriate mathematical model is crucial and completely determines the insight into the actual physical problem that we can obtain by the analysis. The key step in engineering analysis is therefore choosing appropriate mathematical models. These models will clearly be selected depending on what phenomena are to be predicted, and it is most important to select mathematical models that are reliable and effective in predicting the quantities sought.

The most effective mathematical model for the analysis is surely that one which yields the required response to a sufficient accuracy and at least cost $[5,13]$. The chosen mathematical model is reliable if the required response is known to be predicted within a selected level of accuracy measured on the response of the very comprehensive mathematical model. These objectives have been achieved in this work.

Finally, from a practical point of view, the best agreement among the standards and the FEM results corresponds to the NBE-AE Spanish rule. However, some local effects in the transition region from the upstream lateral wall to the roof are observed. This phenomenon is not taken into account for the remaining standards and it must be 
addressed in the design of the building envelope, due to the important local suction effects.

\section{Acknowledgments}

The authors express deep gratitude to the GICONSIME Research Group at Oviedo University for their useful assistance as well as the student Rubén Bermejo Garcia for his valuable collaboration. This work was financed by the Spanish Ministry of Science and Innovation through the Research Project BIA2012-31609, the FICYT PC-10-33 project and the Gijon City Council through the SV-13-GIJON-1.7 project. Besides we would also like to thank Swanson Analysis Inc. for the use of ANSYS University program and Workbench simulation environment.

\section{References}

[1] K.-J. Bathe, Finite Element Procedures, Prentice Hall, New Jersey, 1998.

[2] S.C. Brenner, L.R. Scott, The Mathematical Theory of Finite Element Methods, Springer, New York, 2007.

[3] R.D. Cook, D.S. Malkus, M.E. Plesha, R.J. Witt, Concepts and Applications of Finite Element Analysis, Wiley, New York, 2001.

[4] J.J. del Coz Díaz, P.J. García Nieto, F.J. Suárez Domínguez, Numerical analysis of pressure field on curved self-weighted metallic roofs due to the wind effect by the finite element method, J. Comput. Appl. Math. 192 (1) (2006) 40-50.

[5] O.C. Zienkiewicz, R.L. Taylor, The Finite Element Method: Solid and Fluid Mechanics and Non-linearity, McGraw-Hill Book Company, London, 1991.

[6] P.A. Davidson, Turbulence: An Introduction for Scientists and Engineers, Oxford University Press, London, 2004.

[7] D.C. Wilcox, Turbulence Modeling for CFD, D C W Industries, New York, 2006.

[8] K. Hanjalic, B. Launder, Modelling Turbulence in Engineering and the Environment: Second-Moment Routes to Closure, Cambridge University Press, New York, 2011.

[9] L. Tao, M. Ramakrishna, K.R. Rajagopal, Turbulence modeling from a new perspective, Nonlinear Analysis: Real World Applications 11 (1) (2010) 39-54.

[10] M. Wolfshtein, Some comments on turbulence modelling, Int. J. Heat Mass Tran. 52 (17-18) (2009) 4103-4107.

[11] J.J. del Coz Díaz, P.J. García Nieto, J.A. González Pérez, A. Martín Navarro, Numerical analysis of the pressure field on curved and open self-weighted metallic 
roofs due to the wind effect by the finite volume method, Appl. Math. Comput. 209 (1) (2009) 31-41.

[12] S.B. Pope, Turbulent Flows, Cambridge University Press, New York, 2000.

[13] J.N. Reddy, D.K. Gartling, The Finite Element Method in Heat Transfer and Fluid Dynamics, CRC Publishers, New York, 2000.

[14] G.K. Batchelor, An Introduction to Fluid Dynamics, Cambridge University Press, New York, 2007.

[15] C.A.J. Fletcher, Computational Techniques for Fluid Dynamics, Springer-Verlag, New York, 2001.

[16] C. Hirsch, Numerical Computation of Internal and External Flows, ButterworthHeinemann, New York, 2007.

[17] J.H. Ferziger, M. Peric, Computational Methods for Fluids Dynamics, New York, Springer-Verlag, 2001.

[18] J. Blazek, Computational Fluid Dynamics: Principles and Applications, Elsevier Science, Amsterdam, 2006.

[19] J.J. del Coz Díaz, P.J. García Nieto, J. Ordieres Meré, A. Bello García, Computer simulation of the laminar nozzle flow of a non-Newtonian fluid in a rubber extrusion process by the finite volume method and experimental comparison, J. Non-Cryst. Solids 353 (8-10) (2007) 981-983.

[20] J.J. del Coz Díaz, P.J. García Nieto, A. Bello García, J. Guerrero Muñoz, J. Ordieres Meré, Finite volume modeling of the non-isothermal flow of a non-Newtonian fluid in a rubber's extrusion die, J. Non-Cryst. Solids 354 (47-51) (2008) 5334-5336.

[21] B.E. Launder, B. Spalding, Mathematical Models of Turbulence, Academic Press, New York, 1972.

[22] F. Ilinca, J.-F. Hétu, D. Pelletier, A unified finite element algorithm for twoequation models of turbulence, Comput. Fluids 27 (3) (1998) 133-166.

[23] C. Johnson, Numerical solution of partial differential equations by the finite element method, Cambridge University Press, New York, 1987.

[24] B.E. Launder, CFD for aerodynamic turbulent flows: progress and problems, Aeronaut. J. 104 (2000) 337-345. 
[25] H. Grotjans, F. Menter, Wall functions for general application CFD codes, in: ECCOMAS 98, Proceedings of the 4th European Computational Fluid Dynamics Conference, John Wiley \& Sons, 1998, pp. 1112-1117.

[26] A.J. Lew, G.C. Buscaglia, P.M. Carrica, A note on the numerical treatment of the $k$-epsilon turbulence model, Int. J. Comput. Fluid D. 14 (2001) 201-209.

[27] C.K.G. Lam, K. Bremhorst, A modified form of the $k$-epsilon model for predicting wall turbulence, J. Fluid Eng-T. ASME 103 (1991) 183-205.

[28] E. Madenci, I. Guven, The Finite Element Method and Applications in Engineering using ANSYS®, Springer-Verlag, New York, 2007. 


\section{Table 1}

Value of $n$ for different types of ground Type of ground

Smooth (sea, sand, etc.) $0.10-0.13$

Moderately rough (grass, cereal country,

$0.13-0.20$ rural regions)

Rough (forest, neighbourhoods)

$0.20-0.27$

Very rough (cities, high buildings) 




Fig. 1. Actual view of a curved self-weighted metallic roof. 


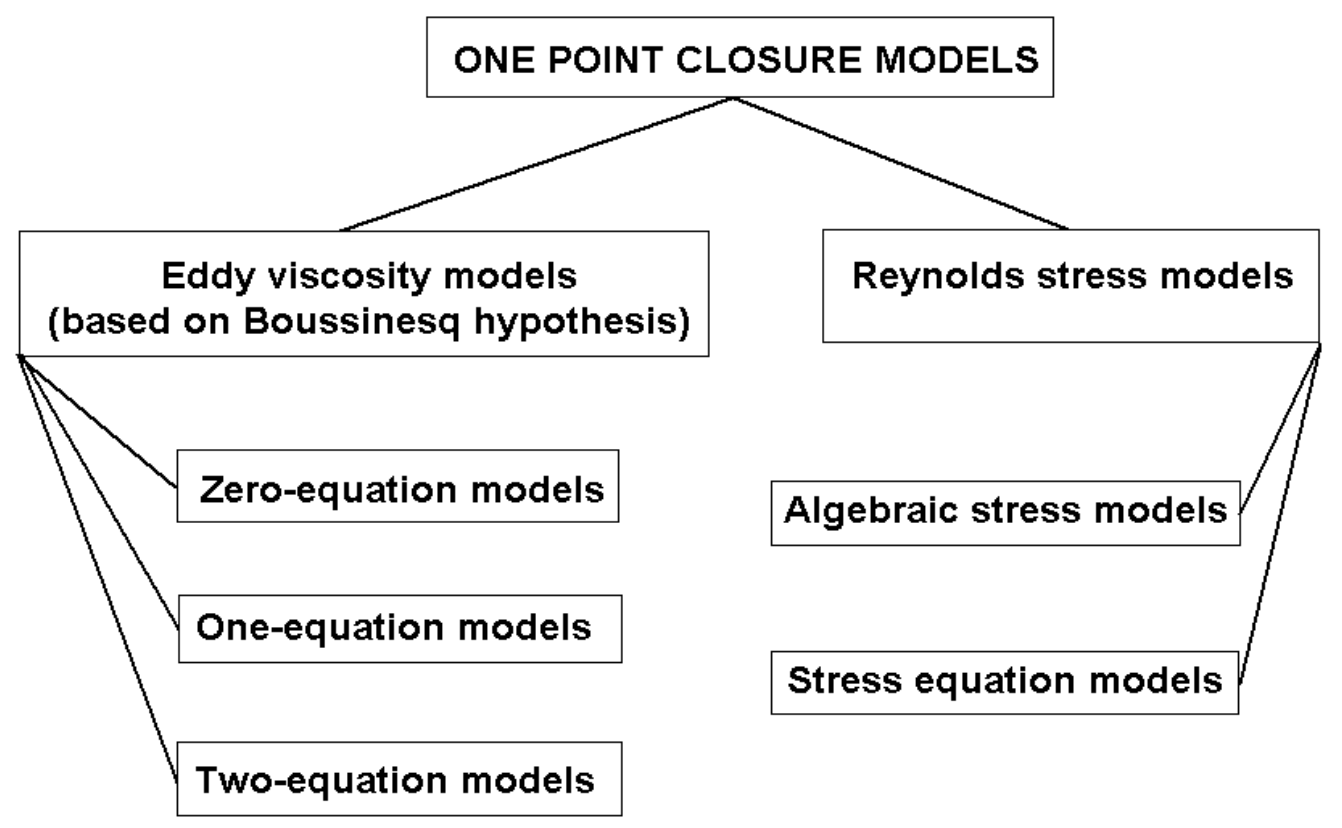

Fig. 2. A classification scheme for the turbulent one point closure models. 


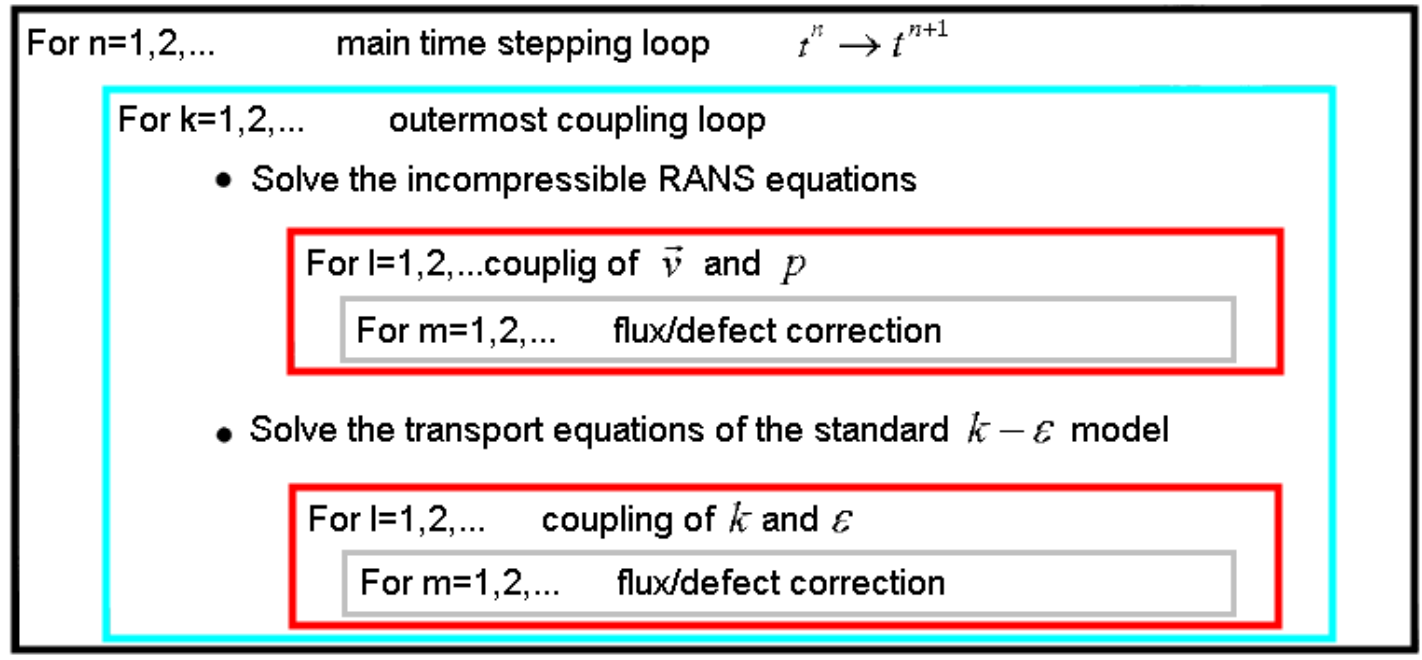

Fig. 3. Hierarchy of nested loops for the iterative solution process. 


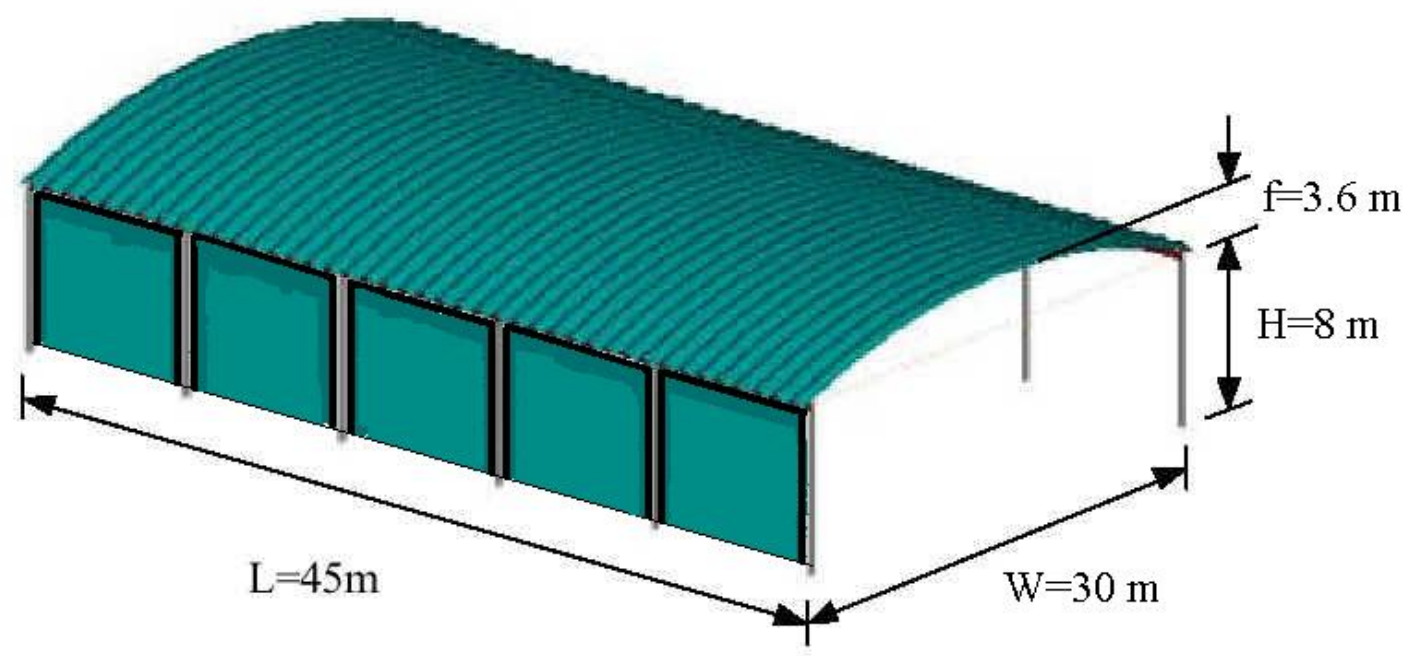

Fig. 4. Dimensions of the roof: length $L=45 \mathrm{~m}$; width $W=30 \mathrm{~m}$; height $H=8 \mathrm{~m}$; arrow and $f=3.6 \mathrm{~m}$. 


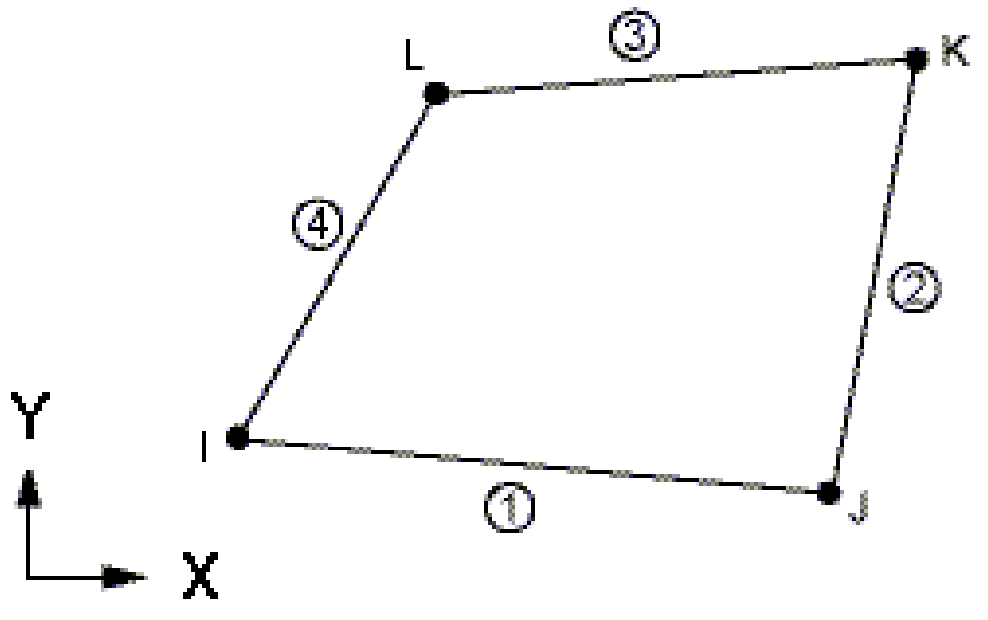

Fig. 5. Finite element FLUID 141 [28] used in the numerical simulation of laterally closed industrial buildings with curved metallic roofs by FEM. 


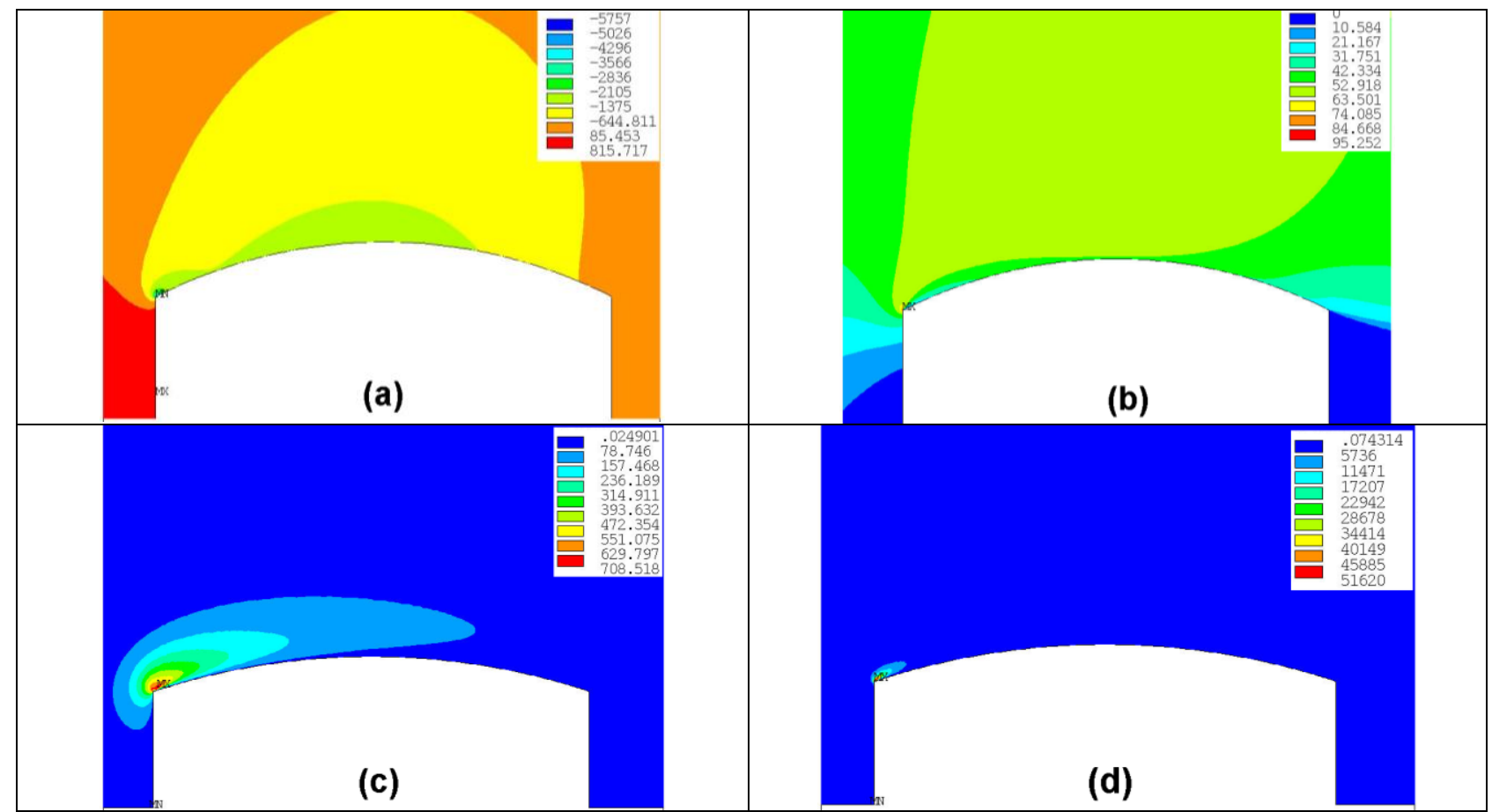

Fig. 6. Numerical contour lines: (a) pressure $(\mathrm{Pa})$ for $v_{r e f}=40 \mathrm{~m} / \mathrm{s}$; (b) velocity $(\mathrm{m} / \mathrm{s})$ for $v_{r e f}=40 \mathrm{~m} / \mathrm{s} ;(\mathrm{c})$ turbulent kinetic energy, $k(\mathrm{~J})$ and $(\mathrm{d})$ turbulent dissipation rate, $\varepsilon$ (J). 


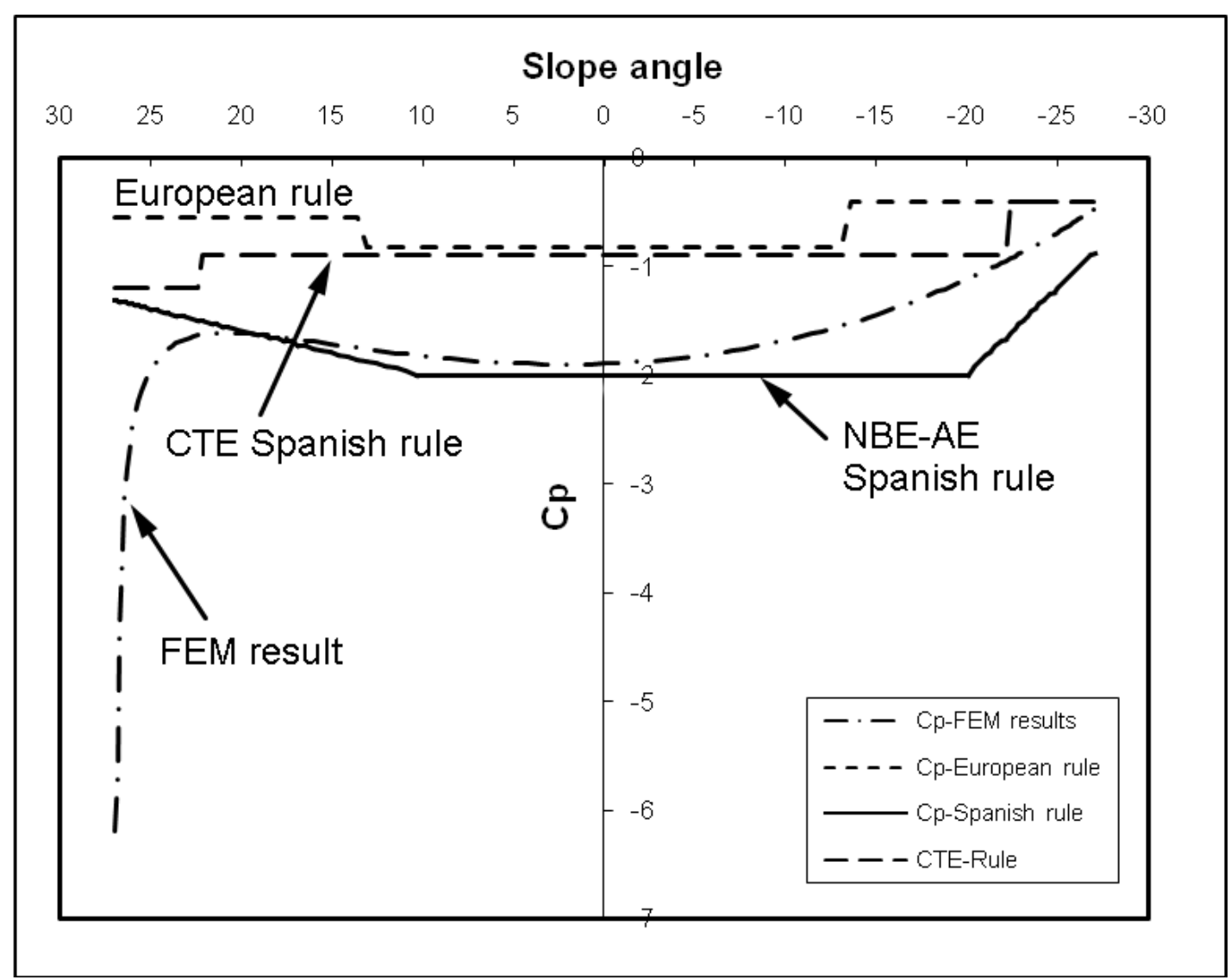

Fig. 7. Pressure coefficient $C_{P}$ obtained by FEM and the estimated ones by the Standard rules $[4,11]$. 